\title{
Clinicopathological Studies in African Catfish (Clarias gariepinus) Affected By Ammonia Toxicity
}

\author{
Abdullah, O.A.M. ${ }^{1}$, Mona M. Abdel-Wahab ${ }^{2}$, Amina A. Dessouki ${ }^{3}$, \\ Haidy G. Abdel-Rahman ${ }^{1}$, Asmaa F. Ibrahim ${ }^{4}$. \\ ${ }^{1}$ Dept. of Clinical Pathology, Faculty of Veterinary Medicine, Suez Canal \\ University. \\ ${ }^{2}$ Animal Health Research Institute, Ismailia. \\ ${ }^{3}$ Dept. of Pathology, Faculty of Veterinary Medicine, Suez Canal University. \\ 4-Directorate of Veterinary Medicine.
}

\begin{abstract}
:
A total number of 60 Clarias gariepinus fish obtained from Ismailia governorate and its tributaries were collected from three locations. The fish were divided into three main groups, (group A) from ElTeraa, (group B) from El-Berkaa, (group C) from El-Rashah. These locations derived from Mohamed Ali channel which derived from River Nile. The fish and water of control group were obtained from central laboratory for Aquaculture Research, El-Abbassa, AboHamad, Sharqia, Egypt. Water analysis of the examined polluted locations revealed high level of ammonia. Serum biochemical examinations revealed hypoproteinemia, hypoalbuminemia and hypoglobulinemia with increase in serum ALT, AST, total bilirubin, direct bilirubin, indirect bilirubin, glucose, urea, creatinine and serum ammonia level in the three groups compared with control one.
\end{abstract}

Key words: ammonia, biochemistry, glucose, protein, ALT, AST, Clarias gariepinus.

\section{Introduction:}

Fish and other aquatic organisms are exposed to great varieties of pollution that have found their way into water in the form of sewage, industrial and agricultural wastes. Many authors had studied the effect of different types of pollutants on fish. Fish production should be increased in Egypt to meet the demand of the increasing population. Several problems face fish production in Egypt. Among these problems are the most tropical species die via low water quality because of pollution with ammonia (Harris et al, 1998).

Ammonia is the principal nitrogenous waste product of fish that represents $60 \%$ to $80 \%$ of nitrogenous excretion of fish (Salin and Williot, 1991). It is also, the main nitrogenous waste material excreted by gills in addition to urea and amines and an end product of 
the protein catabolism (De Croux et al, 2004). Ammonia is toxic, not only to fish but also to all aquatic animals (Harris et al, 1998), especially in pond aquaculture at low concentrations of dissolved oxygen (Alabaster et al, 1983).

Ammonia accumulates to toxic levels; fish cannot extract energy from feed and will fall into a coma and die (Hargreaves and Tucker, 2004). Ammonia tends to block oxygen transfer from the gills to the blood and can cause both immediate and long term gill damage (Joel and Amajuoyi, 2010). Also, it can cause impairment of cerebral energy metabolism, damage to gills, liver, kidneys, spleen and thyroid tissue in fish, crustaceans and mollusks (Smart, 1978).

This work was conducted to study the harmful effects of ammonia toxicity on the African Catfish Clarias gariepinus in three different locations by evaluating: The biochemical analysis, water analysis study, the histopathological alterations induced by ammonia toxicity.

\section{Materials and Methods:-}

Fish

This study was carried out on Catfish Clarias gariepinus belonged to Ismailia Governorate \& its tributaries over three months period from first of May 2014 till end of July 2014. A total number of 60 C.gariepinus with an average body weight $400 \pm 50 \mathrm{~g}$ were collected from three locations \{El-Teraa- El-
Berka- El-Rashah\} derived from Mohamed Ali channel which derived from River Nile. The fish were devided into three main groups according to the site they obtained from. The first group (group A) collected from El-Teraa including 15 fish. The second group (group B) collected from El-Berka including 15 fish. The third group (group C) collected from El-Rashah including 15 fish., each group was subdivided into three subgroups each contain 5 fish according to time they obtained. The fish and water of control group were collected from Central Laboratory for Aquaculture Research, ElAbbassa, Abo-Hamad, Sharqia, Egypt including 15 fish. The fish were immediately transported alive in sterile bags to the lab of Clinical Pathology Dept., Faculty of Vet. Medicine, Suez Canal University.

\section{Water}

Water samples were collected from the three locations at the same time of collection of fish. Water (2 Litre) was collected $50-80 \mathrm{~cm}$ below the water surface in bottles. Water samples were kept in an ice box and immediately transported to the lab of Animal Hygiene, Zoonoses and Animal Behaviour Dept., Faculty of Vet. Medicine, Suez Canal University to examine the physicochemical characteristics.

\section{Blood sampling:-}

The blood was collected from the caudal blood vessels. The blood was left in a plain centrifuge tube without anticoagulant in order to 
clot and centrifuged at $5000 \mathrm{rpm}$ for $5 \mathrm{~min}$ at room temperature, the supernatant serum collected and stored at $-20{ }^{\circ} \mathrm{C}$ in screw epindorph tubes until used for serum biochemical analysis.

Serum

biochemical examinations:-

ALT and AST were determined according to Reitman and Frankel (1957), bilirubin was determined according to Kaplan (1984), total protein was determined according to Henry (1964), albumin was determined according to Drupt (1974), globulin was determined according to Coles (1974), glucose was estimated according to Trinder (1969), urea was determined according to Reiss et al., (1965), creatinine was estimated according to Henry et al., (1974), serum ammonia was estimated by turbidmetry using Coppas 8000. All kits used in this study were obtained from BIO-Merieux (Brains / France) and TichoDiagnostic (Sees, France).

Water analysis:-

Ammonium was determined by using UV screening spectrophotometric method according to $\boldsymbol{A P H A}$ (1998), toxic (unionized) ammonia was calculated using Emerson et al., (1975).

Histopathological examination:-

Tissue specimens from the different organs (gills, liver, kidneys and spleen) of fish were collected and immediately fixed in $10 \%$ formalin solution for $48-72 \mathrm{~h}$. according to Drury and Willington (1980).

Table 1 : Experimental design

\begin{tabular}{|c|c|c|c|c|c|}
\hline $\begin{array}{c}\text { Groups } \\
\text { Time of } \\
\text { Collection }\end{array}$ & Control & $\begin{array}{c}\text { Group A } \\
\text { (El-Teraa) }\end{array}$ & $\begin{array}{c}\text { Group B } \\
\text { (El-Berka) }\end{array}$ & $\begin{array}{c}\text { Group C } \\
\text { (El-Rashah) }\end{array}$ & Total \\
\hline $\mathbf{1}^{\text {st }}$ month & 5 fish & 5 fish & 5 fish & 5 fish & 20 fish \\
\hline $\mathbf{2}^{\text {nd }}$ month & 5 fish & 5 fish & 5 fish & 5 fish & 20 fish \\
\hline $\mathbf{3}^{\text {rd }}$ month & 5 fish & 5 fish & 5 fish & 5 fish & 20 fish \\
\hline Total & 15 fish & 15 fish & 15 fish & 15 fish & 60 fish \\
\hline
\end{tabular}

\section{Results and Discussion:}

The presence of any substance in the water produces changes in their quality which are not always favorable for development and survival of aquatic organisms. When the water quality is affected by toxicant, any physiological changes will be reflected in the values of one or more of the hematological, biochemical and histopathological parameters 
(Adham 2002 and Ishikawa et al, 2007).

Of all the water quality parameters that affect fish, ammonia is the most important after oxygen, especially in semi intensive systems. Ammonia is toxic not only to fish but also to all aquatic animals. Ammonia causes stress and damage to gills and other tissues, even in small amounts (de Oliveira et al., 2012).

In our study, results showed that ammonia level is increased in the three treatments where the highest level was obtained at the third month of collection in El-Berka. Our results are considered higher than the acceptable limits as recommended by Bhatnagar and Singh (2010) who reported that the maximum tolerance level of ammonia for most fish was about $0.1 \mathrm{mg} / \mathrm{L}$ of unionized ammonia $\left(\mathrm{NH}_{3}\right)$. Also, Buttner (1993) reported that ammonia must be limited between 0.2-2.0 mg/L. Yang (1999) concluded that the tolerable level of ammonia for fish culture is $1.2 \mathrm{mg} / \mathrm{L}$. EPA (1998) reported that water with concentrations of less than $0.020 \mathrm{mg} / \mathrm{L}$ unionized ammonia is considered safe for fish reproduction. While Muir et al (2000) recommended that ideal $\mathrm{NH}_{3}$ level for tilapia should be below 0.2 $\mathrm{mg} / \mathrm{L}$.

Biochemical profiles of blood can provide important information about the internal environment of the organism. The role of blood enzymes in monitoring and detecting stress or disease has led to a growing concern in using them as biochemical indicators to trace environmental pollutants (Adham et $a l$, 1999). Data of C. gariepinus in our study revealed that the activities of serum enzymes (ALT and AST) were significantly elevated in response to exposure to high level of ammonia concentrations, with a positive correlation between ammonia concentration and enzyme level elevation, as AST increased than normal value. KrajnovicOzretic and Krajnovic-Ozertic (1992) recorded elevated activities of ALT in the plasma of adult gray mullets Mugilavratus Risso exposed to acute concentrations of phenol and cyanide. Increased level of ALT and AST in common carp after exposure to ammonia toxicity may be due to the loss of Kreb's cycle with the result that these enzymes compensate by providing alpha ketoglutarate (Chatty et al, 1980 and Salah El-Deen 1999). The observed changes could be also due to generalized organ system failure due to the effect of ammonia toxicity.

Bilirubin is a metabolic waste product which formed from the breakdown of erythrocytes. In our study, there was increase in total and direct bilirubin which are indicator for cholestasis and pathological alterations of the biliary flow (Lalitsingh et al, 2010). There was increase in direct and indirect bilirubin in the serum which is indicators for 
hepatocellular jaundice caused by ammonia toxicity (Coles, 1986). Another possible reason may be a metabolic disturbance in liver involving defective conjugation and/or excretion of bilirubin. The bilirubin route of elimination is perhaps most important contributing source to the excretion of xenobiotics, but is of primary importance for the excretion of the animal's metabolites. Since the liver encounters nutrients, environmental toxicants and waste products, within this framework, it extracts the environmental toxicants and waste products to prevent their circulation to other parts of the body (Cheesborough, 1992).

One of the important functions of plasma/serum protein is the maintainance of osmotic balance between the circulating blood and tissue fluids (Harper et al, 1979). The influence of toxicants on the total protein concentration of fish has also been taken into consideration in evaluating the response to stressors and consequently the increasing demand for energy. Concerning serum protein level in our study, the results showed that there was decrease in total protein, albumin and globulin level. These results may be attributed to the severity of the stressor, which causes osmotic imbalance. This result is in agreement with Elbealy (2012) and Alkahem et al, (1998) who attributed the reduction in the proteins to its conversion to fulfilling an increased energy demand by fish to cope with detrimental conditions imposed by a toxicant. This result was in contrary with Seham (2013) who attributed the increase in total protein, albumin and globulin to the changes taking place in serum globulin metabolism or to the input of different pollutants.

The blood glucose was the most sensitive parameter in detecting the sublethal stress response. The serum glucose level was elevated in our study. This result may be due to increase in plasma concentration of catecholamines and corticosteroids as stress response of fish subjected to environmental alterations (Tayel et al, 2008). Glucose increased to cope with stress and maintain homeostasis (Ackerman et al, 2006). Under stress conditions, hypothalamo-pituitaty interregnal axis elevated blood cortisol which in turn leads to glycogenolysis, lypolysis and gluconeogenesis to provide energy. The reported hyperglycemia may be due to withdrawn of water from blood to muscles to overcome the pollution present in water (Massoud et al, 1973) and/or due to the breakdown of glycogen in liver as a result of water pollution (Haggag et al, 1993). Also, this hyperglycemia may be caused by enhanced glycogen breakdown in liver, probably because of anaerobic stress and/or the discharges of various types of wastes. This result is in contrary with Buckley et al, 
(1979) who observed that blood glucose diminished whereas liver glycogen stores increased in Coho salmon exposed for 91 days to 3 , $16,47 \mathrm{mg} \mathrm{N} / \mathrm{L} \mathrm{NH}_{4} \mathrm{CL}$.

Most teleost fish is obligate ammonioteles excreting the bulk 75 - $90 \%$ of their waste nitrogen as ammonia (Hamdy and Poxton, 1993), together with only small amounts ( $5-15 \%)$ of urea produced by uricolysis (Wood, 1993). Urea occurs in natures as the major nitrogen containing end product of protein metabolism by vertebrates, which excrete urea in urine. Creatinine is a nitrogenous waste product, which is synthesized in the body at a fairly constant rate from creatine. The serum urea and creatinine levels in our study were increased in ammonia exposed fish. This may be attributed to renal damage which could be due to the toxicity lead to decrease the filtration rate of the kidneys and thus retention of the urea excretion and creatinine. These results are in agreement with Mcdonald and Milligan (1992). Harvey (1997) reported that the creatinine measurement was more indicative and of more diagnostic value in assessment of renal function activities than blood urea level.

African catfish successfully control plasma $\mathrm{NH}_{4}^{+}$concentrations within physiological concentrations over a wide range of water ammonia concentrations that would be lethal to many other fishes. In African catfish plasma total ammonia is predominantly present (84-98\%) as $\mathrm{NH}_{4}{ }^{+}$(Ip et al, 2004). In our study, results showed that there was increase in serum ammonia level. This may be due to that in African catfish, exposure to high water ammonia $\left(\mathrm{NH}_{3}\right)$ initially results in a plasma $\mathrm{NH}_{4}{ }^{+}$peak due to an $\mathrm{NH}_{3}$ influx followed by the onset of $\mathrm{NH}_{3}$ defense mechanisms over time. Our results was in agreement with Knoph and Thorud (1996) who observed that plasma total ammonia level increased linearly with the water total ammonia level in Atlantic salmon. Also, Person et al (1997) observed that blood plasma TAN contents were positively correlated with ambient ammonia concentrations in three batches of turbot Scophthalmus maximus juveniles exposed for 4-6 weeks to constant ammonium chloride solutions.

From the present study, it was concluded that there is a real need to study the interrelationships between the pollution of surface waters by a wide range of chemicals and diseases in natural fish populations, and the processes involved. This represents an important but at present underdeveloped field of scientific research. It is very important that this water quality stressor (ammonia) be monitored regularly and level should be controlled through various management practices when necessary. 
Table 2: Ammonia Level alterations of water obtained from El-Teraa, ElBerka, El-Rashah:

\begin{tabular}{|c|c|c|c|c|}
\hline $\begin{array}{l}\text { groups } \\
\text { Months } \\
\end{array}$ & Control & El-Teraa & El-Berka & El-Rashah \\
\hline \multicolumn{5}{|l|}{$1^{\text {st }}$ month of collection } \\
\hline $1-\quad$ TAN (mg/L) & $0.52 \pm 0.08^{\mathrm{d}}$ & $6.63 \pm 0.01^{\mathrm{a}}$ & $4.89 \pm 0.04^{\mathrm{c}}$ & $5.11 \pm 0.01^{\mathbf{b}}$ \\
\hline 2- UIA-N (mg/L) & $0.012 \pm 0.013^{d}$ & $0.13 \pm 0.001^{\mathrm{a}}$ & $0.08 \pm 0.003^{\mathrm{c}}$ & \\
\hline$\frac{2^{\text {nd }} \text { month of collection }}{1-\mathrm{TAN}(\mathrm{mg} / \mathrm{L})}$ & $0.3 \pm 0.06^{\mathrm{d}}$ & $8.04 \pm 0.04^{\mathrm{a}}$ & $6.23 \pm 0.11^{\mathrm{C}}$ & $7.29 \pm 0.11^{\mathbf{b}}$ \\
\hline 2- UIA-N (mg/L) & $0.006 \pm 0.002^{d}$ & $0.39 \pm 0.01^{\mathrm{a}}$ & & $0.31 \pm 0.02^{b}$ \\
\hline$\frac{3^{\text {rd }} \text { month of collection }}{1-\mathrm{TAN}(\mathrm{mg} / \mathrm{L})}$ & $0.49 \pm 0.14^{\mathrm{d}}$ & $10.18 \pm 0.07^{\mathbf{b}}$ & $13.27 \pm 0.18^{\mathbf{a}}$ & $9.15 \pm 0.18^{\mathbf{c}}$ \\
\hline 2- UIA-N (mg/L) & $0.009 \pm 0.012^{d}$ & $1.52 \pm 0.01^{b}$ & $2.23 \pm 0.03^{\mathrm{a}}$ & $0.91 \pm 0.02^{\mathbf{c}}$ \\
\hline
\end{tabular}

Means in the same row having different letters are significantly different at ( $p \leq$ $0.05)$.

Table 3: Serum biochemical findings of examined C. gariepinus fish at first month of collection from the three different locations (El-Teraa, El-Berka, ElRashah).

\begin{tabular}{|c|c|c|c|c|c|c|c|c|c|c|c|c|c|}
\hline $\begin{array}{l}\text { Param } \\
\text { eters } \\
\text { groups }\end{array}$ & 喪定 & 社运 & & 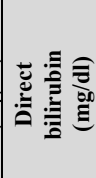 & 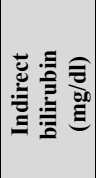 & 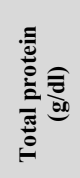 & 言 & $\begin{array}{l}\text { 諳 } \\
\text { 彭 }\end{array}$ & 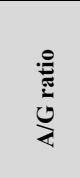 & 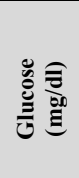 & 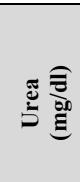 & 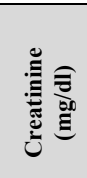 & 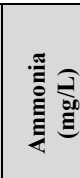 \\
\hline Ü & $\begin{array}{l}\infty \\
\infty \\
0 \\
0 \\
\infty \\
\infty \\
0 \\
-\end{array}$ & 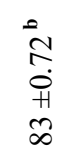 & 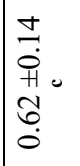 & $\begin{array}{l}\tilde{0} \\
\dot{0} \\
\text { 茾" } \\
\stackrel{0}{0}\end{array}$ & 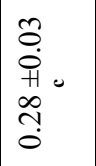 & 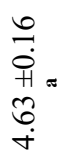 & $\begin{array}{l}7 \\
\stackrel{7}{+} \\
\stackrel{+}{+} \\
\stackrel{4}{i}\end{array}$ & 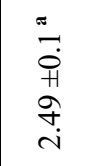 & 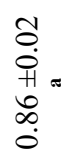 & 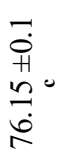 & $\begin{array}{l}\stackrel{\infty}{0}_{0} \\
\stackrel{0}{+1}= \\
\stackrel{2}{a}\end{array}$ & $\begin{array}{l}\stackrel{0}{m} \\
\stackrel{0}{0} \\
\stackrel{+}{+} \\
0\end{array}$ & 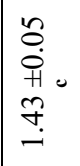 \\
\hline 《 & 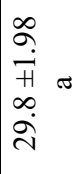 & 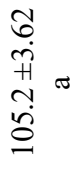 & $\begin{array}{l}\frac{\pi}{0} \\
\overrightarrow{0} \\
+1 \\
\tilde{0} \\
0\end{array}$ & 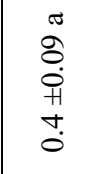 & 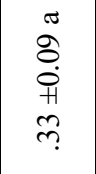 & 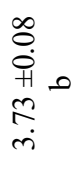 & 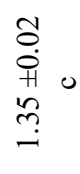 & 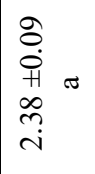 & 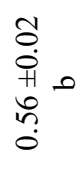 & $\begin{array}{l}0 \\
n \\
+ \\
\dot{H} \\
\dot{0} \\
\infty\end{array}$ & 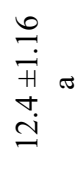 & $\begin{array}{l}\tilde{\delta} \\
0 \\
0 \\
\dot{1} \\
\infty \\
\tilde{0} \\
0 \\
0\end{array}$ & 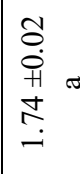 \\
\hline 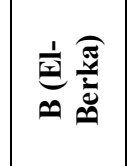 & $\begin{array}{l}\infty \\
\infty \\
\dot{\vec{H}} \\
\infty \\
\dot{\vec{N}} \\
\dot{\vec{N}}\end{array}$ & $\begin{array}{l}\text { त̂ } \\
\text { it } \\
\text { H. } \\
\vec{\Delta} \\
\dot{\infty}\end{array}$ & 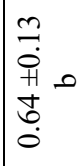 & $\mid \begin{array}{l}n \\
0 \\
0 \\
\text { H } \\
\tilde{n} \\
\tilde{n} \\
0\end{array}$ & 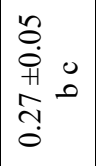 & \begin{tabular}{l}
\multicolumn{1}{c}{} \\
$\dot{+}$ \\
$\dot{+}$ \\
$\alpha$ \\
$\alpha$ \\
$\dot{m}$
\end{tabular} & $\begin{array}{l}\stackrel{n}{0} \\
\dot{+1} \\
\tilde{n} \\
\stackrel{n}{-}\end{array}$ & 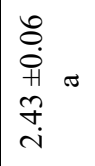 & 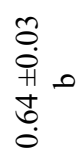 & $\begin{array}{l}\bar{n} \\
0 \\
0 \\
0 \\
0 \\
\dot{R}\end{array}$ & 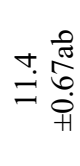 & $\begin{array}{l}\overrightarrow{0} \\
\dot{\oplus} \\
\hat{H} \\
\hat{n}\end{array}$ & 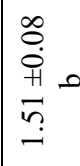 \\
\hline 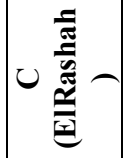 & $\begin{array}{l}\text { ob } \\
\dot{\vec{H}} \\
\overrightarrow{+} \\
\dot{\mathrm{i}}\end{array}$ & 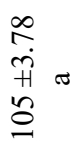 & 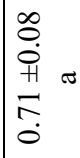 & \begin{tabular}{|l}
$\infty$ \\
0 \\
$\dot{+1}$ \\
0 \\
0 \\
0 \\
0
\end{tabular} & त̂ & 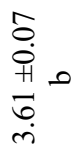 & 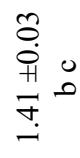 & 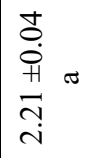 & 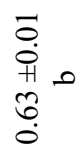 & 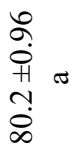 & $\begin{array}{l}\stackrel{0}{0} \\
\stackrel{\leftrightarrow}{1} \\
\infty \\
\stackrel{0}{0}\end{array}$ & $\begin{array}{l}\tilde{\delta} \\
\stackrel{0}{1} \\
\infty \\
\infty \\
0 \\
0\end{array}$ & 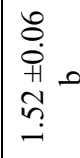 \\
\hline
\end{tabular}

Means in the same column having different letters are significantly different at $(p \leq 0.05)$. 
Table 4: Serum biochemical findings of examined C. gariepinus fish at second month of collection from the three different locations (ElTeraa, El-Berka, El-Rashah).

\begin{tabular}{|c|c|c|c|c|c|c|c|c|c|c|c|c|c|}
\hline $\begin{array}{l}\text { Rarame } \\
\text { ters } \\
\text { Groups }\end{array}$ & 월 & 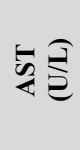 & 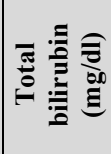 & 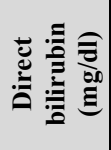 & 施高 & 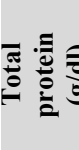 & 言 & 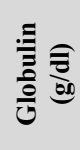 & 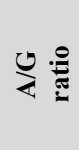 & 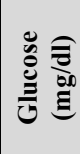 & 总言 & 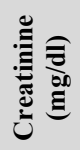 & 氶 \\
\hline 产 & $\begin{array}{l}\ddot{0} \\
\text { Oे } \\
\text { in } \\
\text { iे }\end{array}$ & $\begin{array}{l}5 \\
0 \\
0 \\
0 \\
\text { म1 } \\
\infty\end{array}$ & 它 & 苛 & 范并 & 文 & 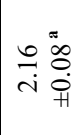 & 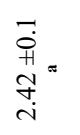 & 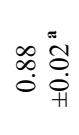 & 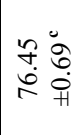 & 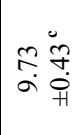 & 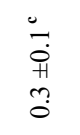 & \\
\hline ব发 & $\begin{array}{l}\text { în } \\
\text { in } \\
\text { 幽 }\end{array}$ & 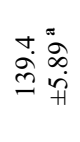 & 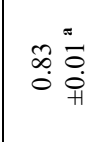 & $\begin{array}{ll}n \\
\text { fó } \\
0\end{array}$ & $\begin{array}{l}\infty \\
m_{0}^{\infty} \\
0 \\
0\end{array}$ & ભ̊ & $\begin{array}{l}\text { on } \\
\dot{0} \\
\dot{H} \\
= \\
=\end{array}$ & 品 & 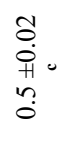 & $\begin{array}{ll}+ \\
\infty \\
\infty\end{array}$ & 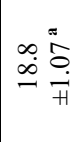 & लें & \\
\hline$\infty \frac{1}{\frac{1}{2}}$ & 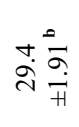 & $\begin{array}{l}\text { aे } \\
\dot{p} \\
\text { a } \\
\sigma\end{array}$ & $\begin{array}{ll}m \\
\dot{0} \\
\dot{0}\end{array}$ & F. & 形 & 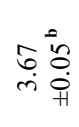 & 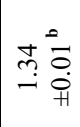 & 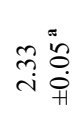 & 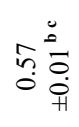 & 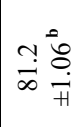 & 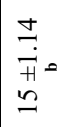 & $\begin{array}{l}\infty \\
0 \\
3 \\
0\end{array}$ & \\
\hline $0 \frac{1}{\sqrt{\frac{\pi}{x}}}$ & 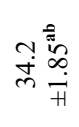 & $\begin{array}{l}\text { to } \\
\text { Oे } \\
\text { In } \\
\Xi\end{array}$ & 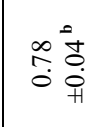 & 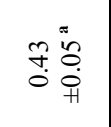 & 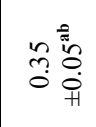 & 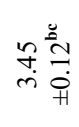 & 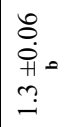 & مُ & 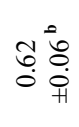 & 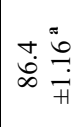 & 菑 & 势点 & $\bar{\sigma}$ \\
\hline
\end{tabular}

Means in the same column having different letters are significantly different at ( $p \leq$ $0.05)$.

Table 5: Serum biochemical findings of examined C. gariepinus fish at third month of collection from the three different locations (El-Teraa, El-Berka, ElRashah).

\begin{tabular}{|c|c|c|c|c|c|c|c|c|c|c|c|c|c|}
\hline 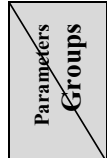 & 월 & 艾全 & 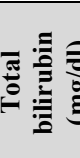 & 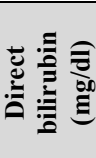 & 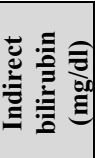 & 嵒 & 言 & 言 & 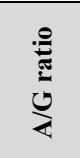 & 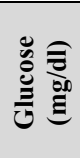 & 总 & 吾。言 & 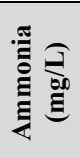 \\
\hline 离 & 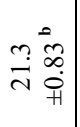 & $\begin{array}{l}\hat{\tilde{O}} \\
\hat{\tilde{H}} \\
\tilde{\infty}\end{array}$ & ت্ّ & 苛 & 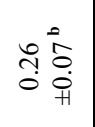 & 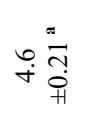 & $\begin{array}{l}\overrightarrow{0} \\
\vec{H} \\
\vec{i}\end{array}$ & 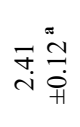 & $\therefore$ & 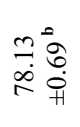 & 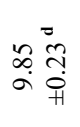 & तે & 喵 \\
\hline & $\begin{array}{l}\tilde{a} \\
\hat{\alpha} \\
+1 \\
\infty \\
\infty\end{array}$ & 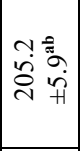 & 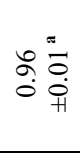 & 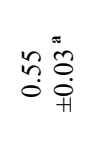 & F. & 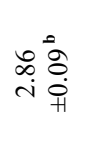 & 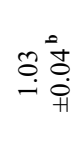 & 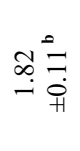 & 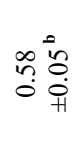 & 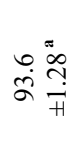 & $\begin{array}{l}\stackrel{\vec{m}}{m} \\
\stackrel{\vec{H}}{\vec{H}} \\
\text { m }\end{array}$ & 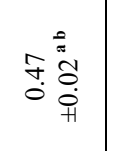 & 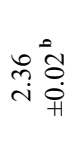 \\
\hline & 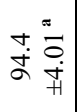 & 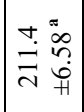 & s. & 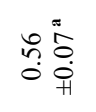 & ţa & त产 & $\begin{array}{l}n: 0 \\
\therefore: 0 \\
0 \\
0\end{array}$ & 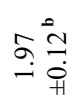 & $\dot{m} \overline{\dot{H}}$ & $\stackrel{+}{\dot{H}} \underset{H}{\stackrel{\pi}{m}}$ & 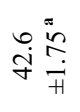 & 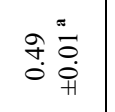 & 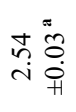 \\
\hline & 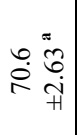 & $\begin{array}{l}\infty \\
\stackrel{\infty}{\tilde{H}}= \\
\vec{a}\end{array}$ & 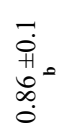 & 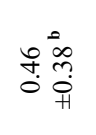 & غ่) & 䓜 & ț & 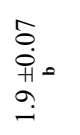 & $\begin{array}{l}n=0 \\
n=0 \\
0 \\
0\end{array}$ & 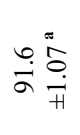 & 苞并 & 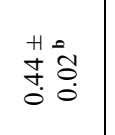 & 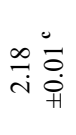 \\
\hline
\end{tabular}

Means in the same column having different letters are significantly different at $(p \leq 0.05)$. 


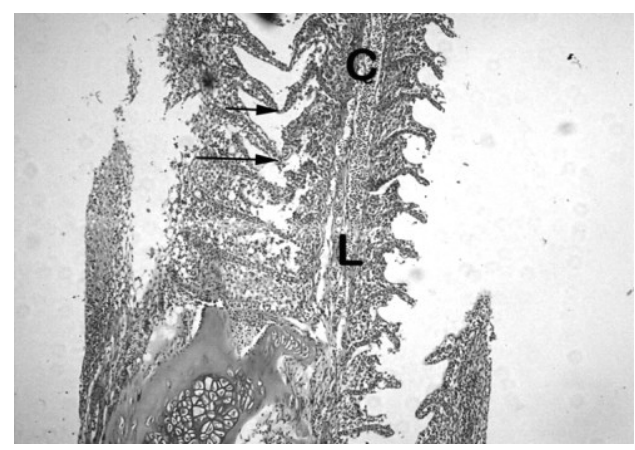

Figure 2: Kidney, catfish exposed to $13.27 \mathrm{mg} / \mathrm{L}$ TAN at El-Berka showing diffuse congestion of blood vessel (arrows) necrotic change of melanomacrophages and degeneration of renal tubules. H\&E. X 100.

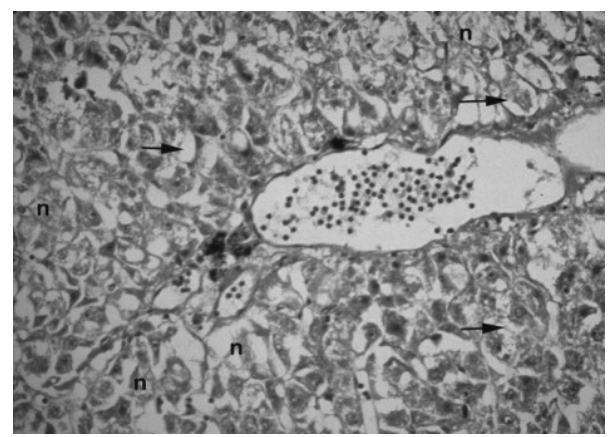

Figure 4: Spleen, catfish exposed to $13.27 \mathrm{mg} / \mathrm{L}$ TAN at El-Berka showing congestion in splenic blood vessel (arrows), hyperactivation of the melanomacrophagecenters (arrow heads) with slight depletion of lymphoid follicles (d). H\&E. X 100.
Figure 1: Gills, catfish exposed to $13.27 \mathrm{mg} / \mathrm{L}$ TAN at El-Berka showed epithelial hyperplasia, adhesion of secondary lamellae (arrows), congestion (C), mononuclear cells infiltration in primary and secondary lamellae (L). H\&E. X 100.

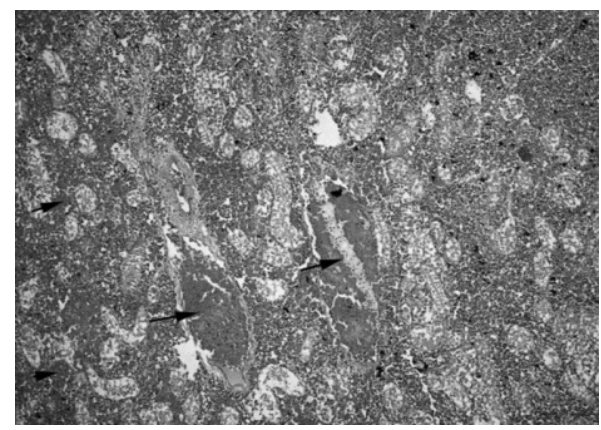

Figure 3: Liver, catfish exposed to $13.27 \mathrm{mg} / \mathrm{L}$ TAN at El-Berka showing vacuolated marked degeneration of hepatocyte (arrows), focal necrosis of some hepatic cells (n), and congestion of hepatic vessels. H\&E. X 400.

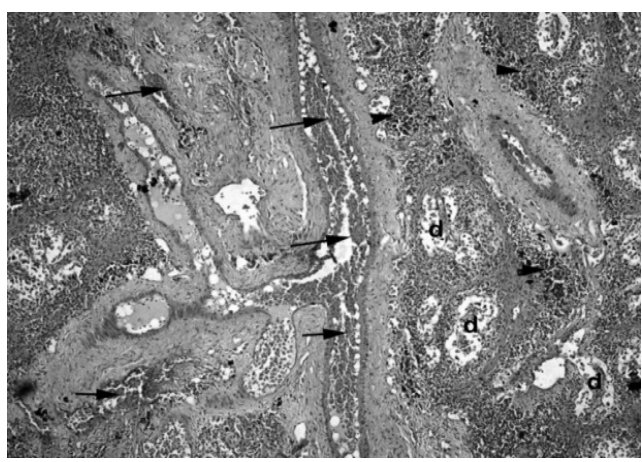




\section{References:}

Ackerman P. A., Wicks B. J., Iwama G. K. and Randall D. J. (2006): Low level of environmental ammonia increase susceptibility to disease in Chinook Salmon smolts. Physiological and Biochemical Zoology, 79 (4): 695-707.

Adham G. K. (2002): Sublethal effect of aquatic pollution in Lake Maryût on the African shaerptooth catfish, Clarias gariepinus ( Burchell,1822). Journal of Applied Ichthyology, 18: 87-94.

Adham K. G., Hassan I. F., Taha N. and Amin T. H. (1999): Impact of hazardous exposure to metals in the Nile and Delta lakes on the catfish, Clarias Lazera. Environ. Monitor. Assess, 54: 107-124.

Alabaster J. S., Shurben D. G. and Malleit M. J. (1983): The acute

lethal toxicity of mixture of cyanide and ammonia to smolts of salmon, Salmo salar $L$. at low concentration of dissolved oxygen. J. Fish Biol., 22: 215- 222 .

Alkahem H. F., Ahmed A. S., AlAkel and Shamusi M. J. K. (1998): Toxicity bioassay and changes in haematological parameters of Oreochromis niloticus induced by Trichlorfon. Arab.Gulf. J. Scient. Res., 16(3): 581-593.

American Public Health Association (A.P.H.A.) (1998): Standard methods for the examination of water and waste water, $20^{\text {th }}$ ed., American Public Health Association, (American
Public Health Association, New York). DC20005-2605.

Bhatnagar A. and Singh G. (2010): Assessment of culture fisheries in village ponds: A study in district Hisar, Haryana, India International Journal of Environmental Research. Iran, 4 (1): 57-64.

Buckley J. A., Whitmore C. M. and Liming D. S. (1979): Effect of prolonged exposure to ammonia on the blood and liver glycogen of Coho-salmon

Oncorhynchus Kisutch. Comp. Biochem. Physiol., 63C: 297-303.

Buttner J. K. (1993): An Introduction to Water Chemistry in Freshwater Aquaculture. North Regional Aquaculture Center NRAC Fact Sheet No: 170.

Chatty C., Naidu Y., Reddy P. and Swan D. (1980): Tolerance limits and detoxification mechanisms in the fish Tilapia mossambica subjected to ammonia toxicity. Indian J. Fish., 27: 177182.

Cheesborough M. (1992): Medical laboratory Manual for tropical countries. Butterworth-Heinemann Ltd., Hakkey Court, Jordan Hill, I: 472-505.

Coles E. H. (1974): Vet. Clin. Path. W.B. Sounders Company, Philadelphia, London, Toronto. 211-213.

Coles E. H. (1986): Text book of veterinary clinical pathology. $4^{\text {th }}$ ed. W.B. Sounders Co., Philadelphia, London. 
De Croux P. M., Julieta and Loteste A. (2004): Lethal effects of elevated $\mathrm{pH}$ and ammonia on juveniles of neotropical fish Colosoma macropomum (Pisces, Caracidae). J Environ. Biol. Jan., 25 (1): 7-10.

De Oliveira E. G., Pinheiro A. B., de Oliveira V.Q., Melo da Silva AR Jr. and de Moraes M. G. (2012): Effects of stocking density on the performance of juvenile pirarucu Arapaima gigas in cages. Aquaculture, 370-371: 96-101.

Drupt F. (1974): Colorimetric method for determination of albumin. Pharm. Bio., 9: 777-779.

Drury R. and Willington E. S. (1980): Carliton histologic technique $5^{\text {th }}$ ed; Oxford.

El-Bealy M. A. A. (2012): Studies on correlation between stress factors and disease problems in Nile Tilapia with a special reference to hematological picture. M. V. Sc thesis Fac. of Vet.Med. Mansoura Univ. Dep. of fish disease and Management.

Emerson K., Russo R., Lund R. and Thurston R. (1975): Aqueous ammonia equilibrium calculations: Effects of $\mathrm{pH}$ and temperature. J. fish. Res. Board Can., 32: 23792383.

EPA (United
Environmental Agency) (1998): Update of ambient water quality criteria for ammonia. USA. United States Environmental Protection Agency, 822-R-98008.52-107.
Haggag A. M., Marie M. A. S., Zaghloul K. H. and Eissa S. M. (1993): Treatment of underground water for fish culture in Abbassa Farm, Sharkia. Bull. Fac. Sci. Cairo Univ., 61: 43-69.

Hamdy R. P. and Poxton M. G. (1993): Nitrogen pollution in mariculture, toxicity and excretion of nitrogenous compounds by marine fish. Rev. Fish Biol. Fish., 3:205-24I.

Hargreaves J. A. and Tucker C. S. (2004): Managing ammonia in fish ponds. SRAC Publication No. 4603: 1-7.

Harper H. A., Rodwell V. W. and Mayes P. A. (1979): Review of physiological chemistry, $17^{\text {th }}$ Ed. Lange Medical publications, Los Altos California.

Harris J. O., Maguire G. B., Edwards S. and Hindrum S. M. (1998): Effect of ammonia on the growth rate and oxygen consumption of juvenile greenlip abalone Haliotis laevigata Donovan. Aquaculture, 160: 259272.

Harvey J. W. (1997): Clinical biochemistry of domestic animals, Fifth edition. Copyright by Academic Press, London, New York, Toronto.

Henry R. J. (1964): Colorimetric determination of total protein In: Clinical Chemistry. Harper and Row Publ., New York, USA,:181.

Henry R. J., Harper R. and Hagerstion R. (1974): Clinical Chemistry, Principles and 
Technique. $2^{\text {nd }}$ ed. HP. Co. Philadelphia.

IP Y. K., Zubaiadah R. M., Liew P. C., Loong A. M., Hiong K. C., Wong W.P. and Chew S. F. (2004): African sharptooth catfish Clarias gariepinus does not detoxify ammonia to urea or amino acids but actively excretes ammonia during exposure to environmental ammonia. Physiol. Biochem. Zool., 77(2): 242-254.

Ishikawa N. M., Tavares R. M. J., Lombardi J. V. and Ferreira C. M. (2007): Haematological parameters in Nile Tilapia, Oreochromis niloticus exposed to sub-lethal concentrations of mercury. Brazilian Archives of Biology and Technology, 50: 619626.

Joel O. and Amajuoyi C. S. (2010): Determination of the concentration of ammonia that could have lethal effect on fish pond. ARPN J. Engineer. Appl. Sci.: 1-4.

Kaplan A. (1984): Bilirubin. Clin. Chem. The C.V. Mobsby Co. St Louis. Toronto. Princeton., 436: 1238-1241.

Knoph M. B. and Thorud $K$. (1996): Toxicity of ammonia to Atlantic salmon Salmo salar L. in sea water-effects on plasma osmolality, ion, ammonia, urea and glucose levels and hematologic parameters. Comp. Biochem. Physiology, 113(4): 375-381.

Krajnovic-Ozretic M. and Krajnovic-Ozertic B. (1992): Detection and evaluation of hepatic intoxication in fish. In: Gabrielides G.
P. (ed.), Workshop on the biological effects of pollutants on marine organisms, Malta, 10-14 Sept 1991. Proceedings of the FAO/UNEP/IOC, MAP Technical Reports, 69: 165175.

Lalitsingh R, Jigar B. and Jagruti P. (2010): Hepatoprotective activity of ethanolic extract of bark of zanthoxylum armatum DC in CCL4 induced hepatic damage in rats. Journal of Ethnopharmacology, 127 (3): 777-780.

Massoud A., Saad H. and Shabana M. B. (1973): Effect of pollution on the blood characteristics of Tilapia Zillii G. Water, Air and Soil Pollut., 2: 171179.

Medonald D. G. And Milligan C. L. (1992): Chemical properties of blood in : Hoar W. S. Randall D. J., Farrell A. P., eds. Fish Physiology Vol. 12 part B, The Cardiovascular System. New York: Academic Press, 56-135.

Muir J. F., Van Rijn J. and Hargreaves J. (2000): Production in intensive and recycle systems. In: Beveridge, M.C.M. and McAndrew, B.J. (eds) Tilapias: Biology and Exploitation. Kluwer Academic Publishers,

Dordrecht/Boston/London, $\quad 405$ 445.

Person-Le Ruyet J., Galland R., Le Roux A. and Chartois $H$. (1997): Chronic ammonia toxicity in Juvenile Turbot Scophthalmus maximus. Aquaculture, 154:155171. 
Reiss D., Muraine J. and Smart G. R. (1978): Investigations Dainciart A. (1965): Transaminases in Serum. Am. J. Clin. Pathology. Bull. Soc. Pharm. Bordeaux, 104-173.

Reitman S. and Frankel S. (1957): Colorimetric determination of glutamic oxaloacetic and glutamic pyruvic transaminases. Am. J. Clin. Pathol., (28): 53-56.

Salah El-Deen M. A. (1999): Toxicological and physiological effects of ammonia on grass carp Ctenopharyngodon idella at different pH levels. Egypt. J. Zool., 33: 219-235.

Salin D. and Williot P. (1991): Acute toxicity of ammonia to Siberian sturgeon Acipenserbaen P. Williot, Ed. A cipenser, Comagref Publ.: 153-167.

Seham Ahmed Ibrahim (2013): hematological and histopathological studies on Tilapia fish Oreochromis niloticus living in the water of Rosetta Branch, River Nile, Egypt. Global Veterinaria, 9- 11 (5): 485496.

on the toxic mechanisms of ammonia to fish gas exchange in rainbow trout Salmo gairdneri exposed to acutely lethal concentrations. J. Fish Biol., 12: 93104.

Tayel S. I., Yacoub A. M. and Mahmoud S. A. (2008): Histopathological and hematological responses to freshwater pollution in the Nile Catfish Clarias gariepinus. J. Egypt. Acad. Soc. Environ. Develop., 9: 43-60.

Trinder P. (1969): Determination of glucose concentration in the blood. Ann. Clin. Biochem, 6-24.

Wood C. M. (1993): Ammonia and Urea Metabolism and Excretion. In the Physiology of Fish (ed., D.H. Evans), Boca Raton, FhCRCPrss: 379-425.

Yang Y. (1999): Modeling growth of Nile tilapia Oreochromis niloticus in a cage-cum-pond integrated culture system. Aquacultural Engineering, 21(2):113-133. 


\section{الملخص العربي}

\section{دراسات باثولوجية اكلينيكية في أسماك القرموط الأفريقى المصابة بتسمم الأمونيا}

أسامة علي عحم عبدالله ـ مني محمد عبدالوهابـ أمينة علي دسوقي- هايدي جلال عبدالرحمن- أسماء

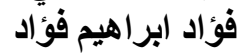

ـتتأثر الأسماك كأي كائن حي بالبيئة المحيطة بها فعندما يحدث خلل في أي من العوامل البيئية

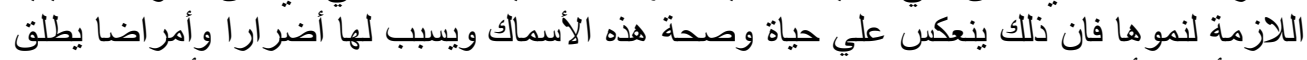

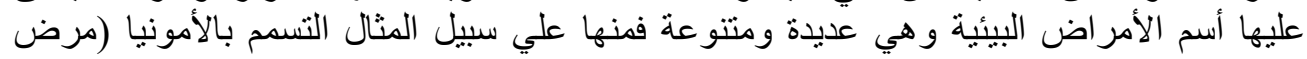

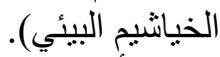

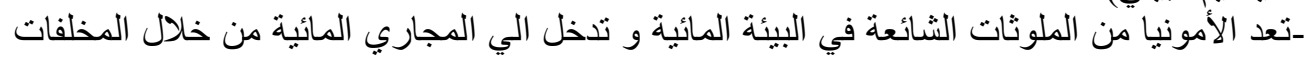

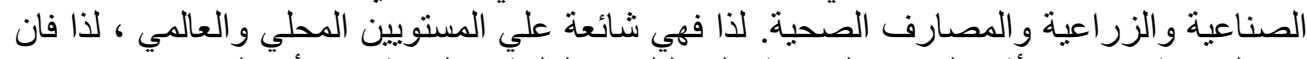

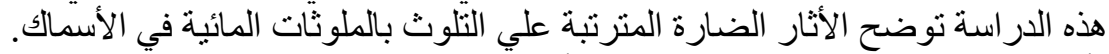

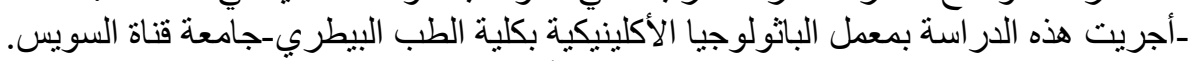

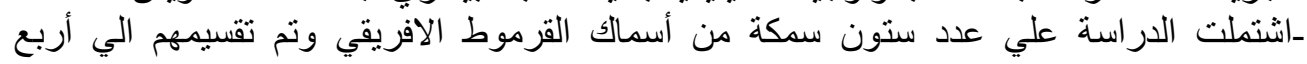

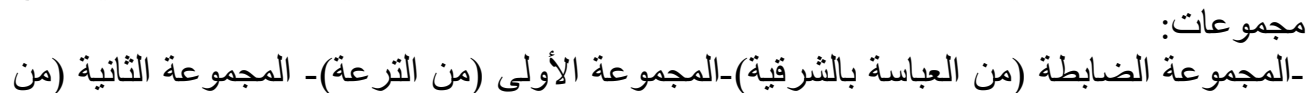

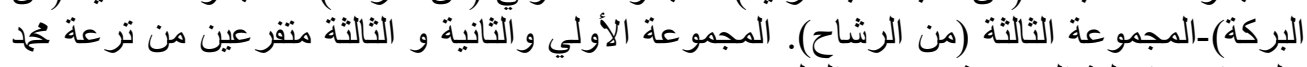

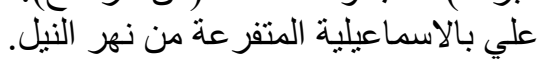

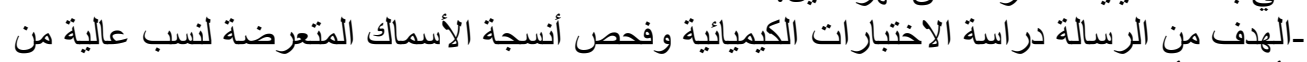
الأمونبا وأسفرت النت النتائج عن الاتي:-

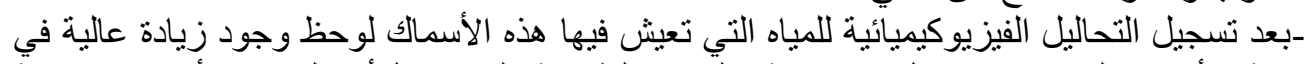

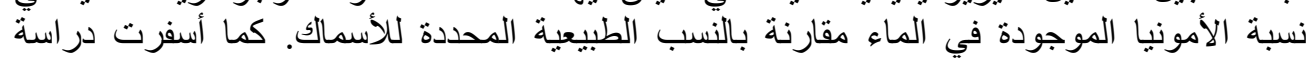

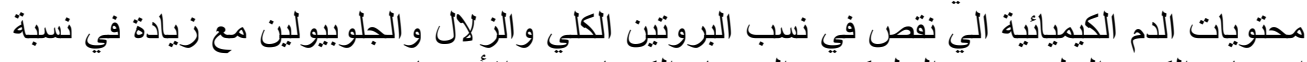

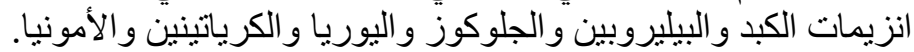

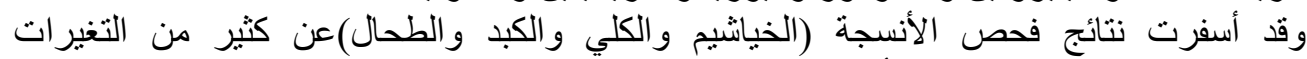

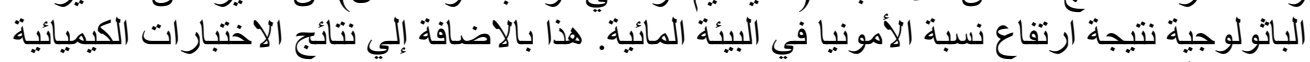

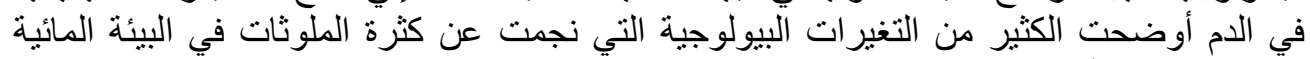
الكائن بها تلك الأسماك محل البحث. 